\title{
Detector simulation of LHAASO-KM2A with Geant4
}

\section{Songzhan Chen * Jing Zhao, Zhe $\mathrm{Li}^{\dagger}$, on behalf of the LHAASO Collaboration}

Key Laboratory of Particle Astrophyics, Institute of High Energy Physics, Chinese Academy of Sciences, Beijing, 100049, China

TIANFU Cosmic Ray Research Center, Chengdu, China

E-mail: chenszeihep.ac.cn

\begin{abstract}
LHAASO is a large hybrid EAS experiment located in at Haizi Mountain, Daocheng, Sichuan province. KM2A is one of the main subarrays of LHAASO mainly for $\gamma$-ray astronomy and cosmic ray physics at energy above $20 \mathrm{TeV}$. The whole KM2A array consists of 5195 electromagnetic detectors (EDs) and 1171 muon detectors (MDs). The detectors construction is underway and a part of KM2A array have been operated since February 2018. The whole array will be finished until year 2021. Detector simulation is the important foundation for estimating detector performance and data analysis. While it is a big challenge to construct the KM2A detector simulation software in the framework of Geant4 due to large number of detector units $(>6000)$, huge coverage $\left(1.36 \mathrm{~km}^{2}\right)$, large altitude difference $(30 \mathrm{~m})$, and huge photons tracing. In this talk, the design of KM2A array simulation code G4KM2A basing on Geant4 is introduced. The process of G4KM2A is optimized in memory consuming to avoid memory overflow. Some simplifications are used to significantly speed up the executing of G4KM2A. A comparison between MC and the part KM2A array data are also presented.
\end{abstract}

36th International Cosmic Ray Conference -ICRC2019-

July 24th - August 1st, 2019

Madison, WI, U.S.A.

*This work is supported by the National Key R\&D Program of China (No. 2018YFA0404201) and the Natural Sciences Foundation of China (No. 11575203, 11635011).

$\dagger$ Speaker. 


\section{Introduction}

The Large High Altitude Air Shower Observatory (LHAASO) [四] is a large hybrid extensive air shower (EAS) array being constructed at Haizi Mountain, Daocheng, Sichuan province, China. It is composed of three subarrays, i.e., $1.3 \mathrm{~km}^{2}$ array (KM2A), $78,000 \mathrm{~m}^{2}$ water Cherenkov detector array (WCDA) and 12 wide-field air Cherenkov/fluorescence telescopes (WFCTA). The whole KM2A array consists of 5195 electromagnetic detectors (EDs) and 1171 muon detectors (MDs). The detectors construction is underway and a part of KM2A array have been operated since February 2018. 1/4 of KM2A will start running in August 2019. The whole array will be finished until year 2021 .

$\mathrm{KM} 2 \mathrm{~A}$ is mainly for $\gamma$-ray astronomy and cosmic ray physics at energy above $20 \mathrm{TeV}$ [四]. EDs could precisely measure the arrival time and density of the particles of cosmic ray or $\gamma$-ray induced shower. This information will be used to reconstruction the direction and energy of the primary particles. Its angular resolution reaches about 0.3 degrees at $100 \mathrm{TeV}$, and the energy resolution is better than $25 \%[$ [B] . MDs mainly detect the muons, which are used for primary component discrimination between gamma-ray and cosmic ray background, or among different cosmic ray nuclei. Due to the powerful $\gamma$-ray/Proton discrimination ability, the sensitivity of KM2A for $\gamma$-ray source is unprecedented at energy above $20 \mathrm{TeV}$ and reach to a flux of $1 \%$ Crab unit at $50 \mathrm{TeV}$ [四]. This will be crucial to identify cosmic ray Pevtron sources [0].

Detector simulation is the important foundation for estimating detector performance and data analysis. When a high energy $\gamma$-ray or cosmic ray enters the atmosphere, it dissipates its energy through interaction with the air molecules and cause a cascade shower. The number of secondary particles arriving at ground depends on the primary particle energy and zenith angle, and also is stochastic dominated. Since the limitation of detector coverage, only a small portion of secondary particles can be recorded by EAS array. This also depends on the response of detector for different secondary particles. Therefore, to estimate the primary information, such as energy, component, flux and so on, of cosmic ray or $\gamma$-ray, the simulation technique must be adopted. Usually, the cascade processes within the atmosphere can be well simulated via the CORSIKA code [四], while, an accurate detector response simulation need to be developed in the framework of Geant4 package [[]]. In this proceeding, the LHAASO-KM2A detector simulation code G4KM2A developed basing on Geant4 package will be introduced.

\section{Detector simulation}

Geant 4 is a toolkit for the simulation of the passage of particles through matter. It provides a battery of tools to describe the geometry and material properties of an experimental setup, handle particle transport through materials and magnetic fields, and simulate particle decay and interactions with detector elements. The detectors of KM2A are constructed as follows.

\subsection{ED detector}

An ED consists of 4 plastic scintillation tiles $(100 \mathrm{~cm} \times 25 \mathrm{~cm} \times 1 \mathrm{~cm}$ each). When a high energy charged particle enter into the scintillation, it will lose energy and excite the scintillation medium 
to produce a large amount of scintillation photons which follows a certain wavelength distribution as shown in Fig.1. Each tile has a coating made by a highly diffusive and reflective fabric of commercial Tyvek to contain the scintillation light inside the volume of tile. A dielectric surface is adopted to treat the interactions of photons with the reflector of Tyvek. The reflectivity is wavelength dependent and the maximum value is set to be $91.5 \%$. The coated tile is covered by 5 -mm-thick lead plate to absorb low-energy charged particles in showers and to convert $\gamma$-rays into electron-positron pairs. Sixteen wavelength-shifting fibers (WLS, $2.7 \mathrm{~m}$ in length and $1.5 \mathrm{~mm}$ in diameter) are embedded in 32 grooves (each $1.8 \mathrm{~mm}$ in depth and $1.6 \mathrm{~mm}$ in width) of each tile to collect scintillation light. The WLS will absorb the scintillation photon and emit photon with a longer wavelength as shown in Fig.1. The absorption length of light within fiber is set to be $2.6 \mathrm{~m}$. In the practical detector, 128 fibers ends from four tiles are bundled together with connecting to a 1.5-inch photomultiplier tube (PMT). While, it is difficult to describe the geometry of fibers from tile to PMT within Geant4 due to the diversity of the 128 fibers. Therefore, an equivalent method is adopted in the simulation with each unbent fiber connecting to a tiny PMT, which is defined as the sensitive detector. Each photon impinging on the PMT surface is counted with a certain probability which depend on the photon wavelength due to the quantum efficiency $(\mathrm{QE})$ of PMT as shown in Figure 1. Figure 2 exhibit the geometry of simulated ED and the transport of photons when a high energy muon pass through.
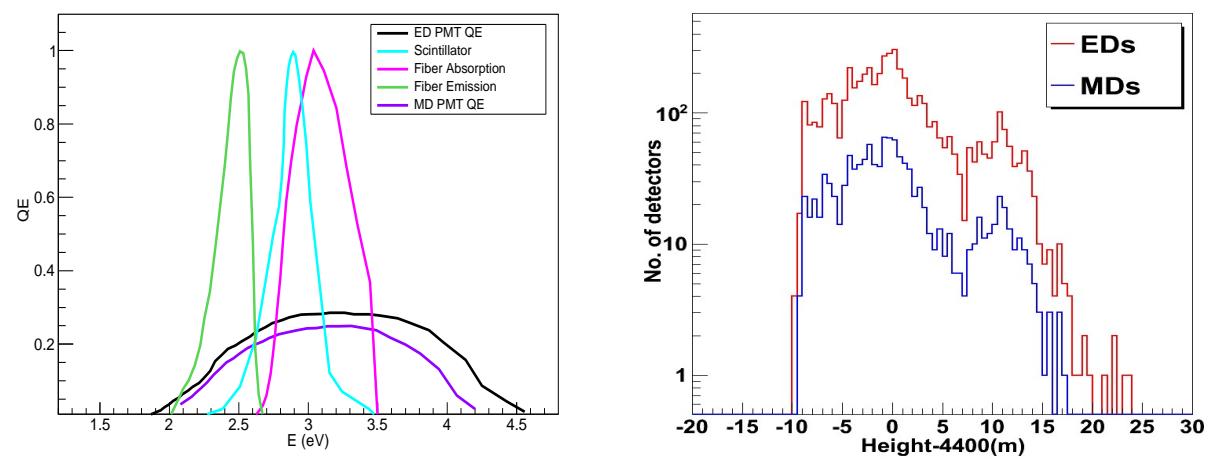

Figure 1: left: The energy distribution of photons emitted by scintillator, absrobed by WLS fiber, emitted by WLS fiber, and the quantum efficiency of ED PMT and MD PMT. Right:The distribution of the altitude of the ED and MD.

\subsection{MD detector}

The MD is pure water Cherenkov detector enclosed within a water bag which is also contained in a cylindrical concrete tank with an inner diameter of $6.8 \mathrm{~m}$ and height of $1.2 \mathrm{~m}$. The absorption length of light in water is wavelength dependent and the maximum value is $150 \mathrm{~m}$. The water bag has an inner coating made by a highly diffusive and reflective fabric of commercial Tyvek to contain the Cherenkov light inside bag. The inner surface coating was simulated similar to the reflector of Tyvek in ED. The reflectivity is wavelength dependent and the maximum value is $97.5 \%$. The 8 -inch PMT is simulated as a hemispheric volume of radius $10.1 \mathrm{~cm}$ immersed in the water volume at the roof center. The PMT is defined as the sensitive detector. Each photon impinging on the 

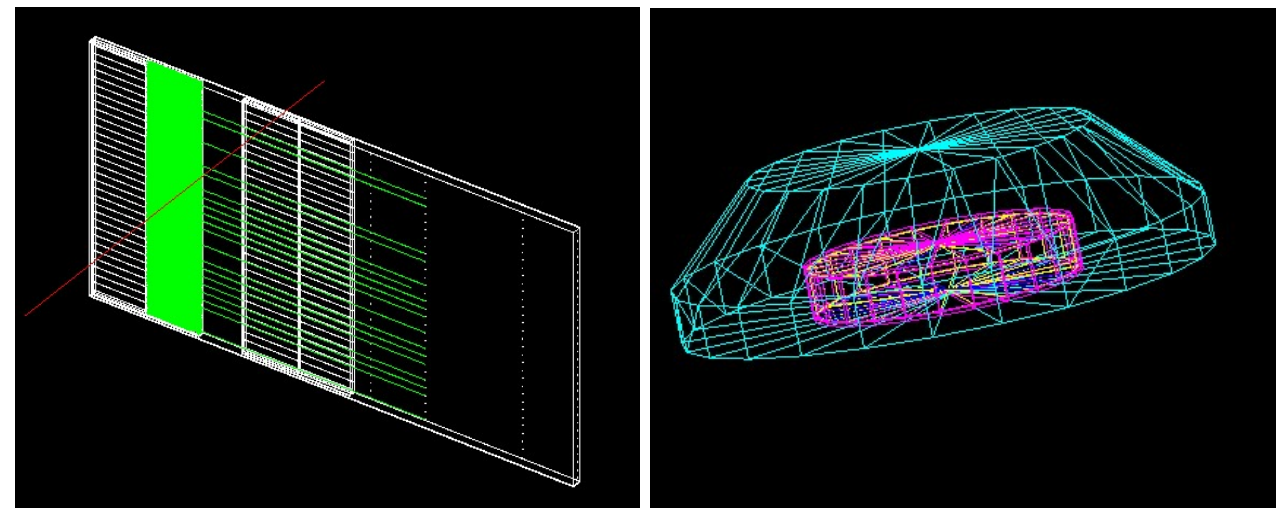

Figure 2: left: Illustration of ED response to a pass through high energy muon. Right: The geometry of the MD unit constructed in the simulation.

PMT surface is counted with a certain probability due to the quantum efficiency (QE) of the PMT as shown in Figure 1. The collection efficiency is set to be $85 \%$. The concrete tank is covered by a steel lid underneath soil. The thickness of overburden soil is $2.5 \mathrm{~m}$. To simplify the simulation, only the soil surround the tank with a diameter of $13.9 \mathrm{~m}$ is constructed as shown in Figure 2. Such a simplification has little impact on the particles with zenith angle less than $71^{\circ}$. It is worth to note that such a simplification could also speed up the executing of simulation since the transport of high energy particles within soil is heavy time consuming.

\subsection{KM2A array}

The whole KM2A array consists of 5195 EDs and 1171 MDs, which spread over an area of $1.3 \mathrm{~km}^{2}$. According to the measurement, the largest altitude difference of the KM2A detectors will be about $35 \mathrm{~m}$ as shown in Figure 1. The detectors will be constructed and merge into the data acquisition system stage by stage. To adapt to this requirement, a flexible strategy is adopted to construct the KM2A array. The simulation code read an external text file which contains three dimension coordination of the EDs and MDs, and then to construct the EDs and MDs according to the coordination. In such a way, it can simulate the KM2A array with any configuration.

\subsection{Speed up methods}

Usually, a large amounts of showers need to be simulated and each shower is comprised of a large amounts of high energy secondary particles. For both ED and MD, there are a large amounts of photons within the detector when one particle pass through. In such a situation, the tracking of photons within the simulation is heavy time consuming which make it hard to be used in practice. Therefore, the simulation procedure must be speeded up.

In fact, both ED and MD adopt PMT to detect the photons, due to the QE of PMT as shown in Figure 1, only 20\% photons impinging on the PMT can be recorded. To reduce the tracking of useless photons, the Cherenkov photons produced in the MD are randomly killed according the QE before tracking. For the scintillation photons produced in ED, the QE is realized with two steps. Firstly, a constant efficiency correspond to the maximum value of QE is applied in the scintillation tile. The residual wavelength dependent efficiency is applied within the WLS fiber after the change 
of photon wavelength. With such an optimization, the speed of the simulation procedure could be improved by a factor about 5 .

Beside above speed up method, a further optional speed up method is also provided. According to the tracking result, only a tiny fraction $(<1 \%)$ of photons produced in ED or MD can propagate to the PMT. There are 32 fibers in each ED scintillation tile, the possibility of photons reaching to the PMT has little position and direction dependence. In such a situation, all the photon tracking within the ED can be killed with being replaced by a simple sampling, and then sampling a random transport time for survival photons according to the tracking result. Figure 3 shows the comparison between tracking result and fast sampling result about the number of photons recorded by PMT for $5 \mathrm{GeV}$ muons simulation. Obviously, they are consistent with each other very well. With such a simplification, the speed of the ED simulation could be improved by a factor about 100 . For the $\mathrm{MD}$, there is only one PMT within a volume of $44 \mathrm{~m}^{3}$, therefore, the possibility of the photons reaching to the PMT is position and direction dependent. A simple direct simplification as that of ED will cause some difference as shown Figure 3. While, if the simplification is applied after 5 times reflection, the possibility will has little position and direction dependence, and the result will be consistent with the tracking result as shown in Figure 3. A random transport time is also sampled for the survival photon according to the tracking result. This simplification could speed up the MD simulation by a factor about 4 .
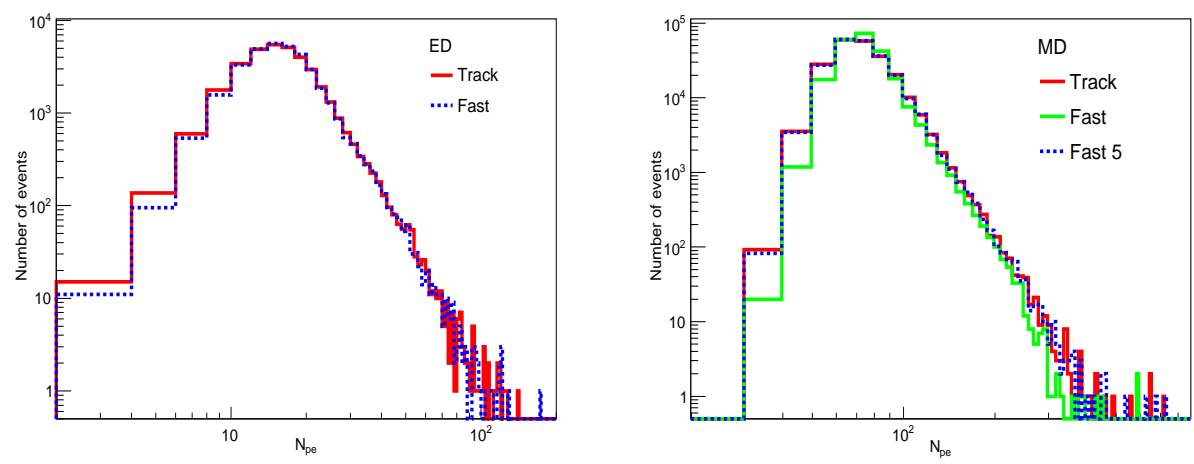

Figure 3: left: The numbers of photons recorded by ED PMT using photon tracking and fast simplification method, respectively. Right: The numbers of photons recorded by MD PMT using photon tracking, direct fast simplification, 5 times reflection+ fast simplification, respectively.

\subsection{Optimization of simulated process}

In the framework of normal Geant4, the particle generator will generate all the particles for one event, than the internal mechanism will track all the particles and their secondary particles. During this process, some computational memory will be consumed to record the particles and their tracking information. Due to the limitation of computational memory size, the memory will overflow if there are too many particles in one event. For the simulation of G4KM2A, the particle generator will read-in a cosmic ray shower produced by CORSIKA, each shower is comprised of a large amounts of high energy secondary particles. Each secondary particle can induce a lot of subordinate particles and photons when enter into the ED or MD. For such a situation, the 
simulation procedure always crash due to memory overflow. To solve this problem, the simulation process flow is adjusted by reading-in the particles of shower one by one in the particle generator. For each secondary particle, it will reach the end of event, and some internal mechanism will release the memory at this step. The Figure 4 shows the simulation process before and after the adjustment. It is worth to note that, the affection of such an adjustment on the speed of simulation execution is ignorable.

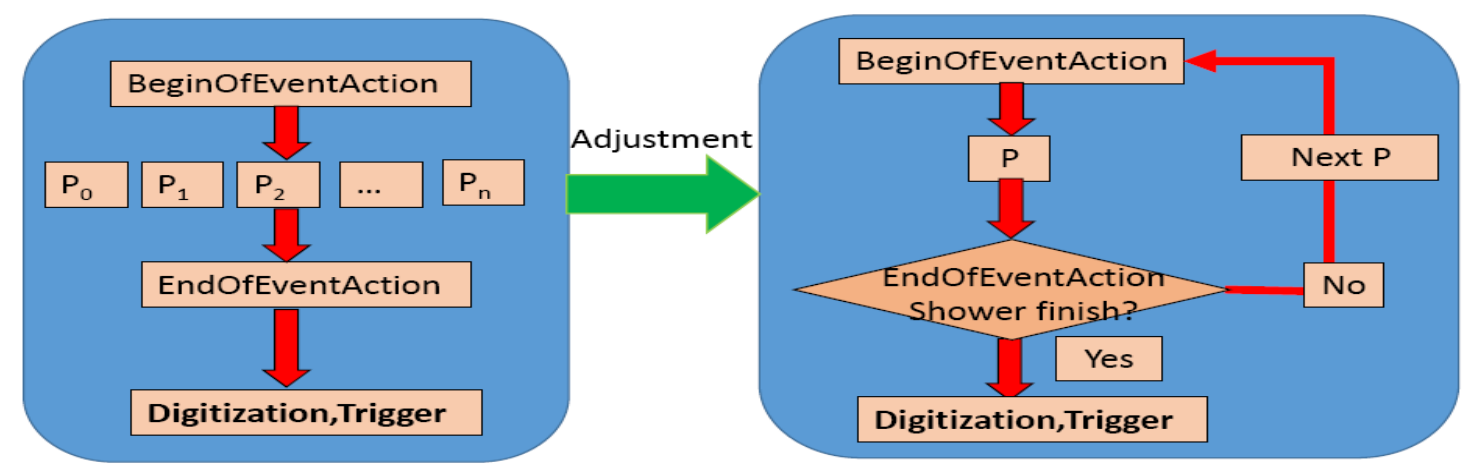

Figure 4: The flow diagram of simulation process before and after adjustment. $\mathrm{P}$ stands for one secondary particle of a shower.

\section{Digitization and trigger Simulation}

In the framework of Geant4, particle transport and interaction within the detector can be realized via the provided tools. The internal mechanism will tracking photon up to the sensitive detector PMT. Then, we need write code to process these photons and convert them to signal similar to the real detected signal. These include digitization, adding noise hits and trigger discrimination.

\subsection{Digitization simulation}

For a real PMT, it converts each incident photon into electrical pulse. In this work, all the recorded photons are converted into electric pulses using a formulaic shape:

$$
A\left(\frac{t}{\tau}\right)^{2} e^{-\left(\frac{t}{\tau}\right)^{2}}
$$

Where $\mathrm{A}$ is the amplitude, and $\tau$ stands for the characteristic time of the shape. This shape is determined from the measurement of single photoelectron pulse via oscilloscope. For the ED PMT, $\tau$ is fixed to be $3.5 \mathrm{~ns}$ and a fluctuation of $50 \%$ of $\mathrm{A}$ is taken into account in this simulation. Beside this, a Gaussian shape fluctuation with $\sigma=1 \mathrm{~ns}$ and fixed $30 \mathrm{~ns}$ delay are added for the transport time from photon to electrical pulse. After this conversion, a time dependent electrical pulse wave for each detector unit is achieved basing on which we digitize wave into hits with TDC for time information and ADC for charge information when the amplitude higher than the setting threshold. According to the characters of electronic devices used by the experiment, the dead time of ED is set to be $16 \mathrm{~ns}$ and $400 \mathrm{~ns}$ for TDC and ADC, respectively. The dead time of MD is set to be 608 ns for both TDC and ADC. 


\subsection{Noise simulation}

For each triggered event, the recorded time window is 10 us. During this period, some random noise hits from single particles, ambient radioactivity, and electronic noise can also be registered. According to the data of partial KM2A array at LHAASO site, noise hit rate of ED unit is about $1.7 \mathrm{kHz}$ and that of MD unit is about $7 \mathrm{kHz}$, which are adopted in this simulation. The arrival times (TDC) of noise hits are sampled with randomly and uniformly distribution. The charges (ADC) are sampled according to the measurement result of partial KM2A array.

\subsection{Trigger logic}

For the KM2A array, only the hits recorded by ED array are used for trigger discrimination. An EAS composed of many secondary particles that can fire multiple detectors in a short time period. At the same time, multiple detectors have a certain possibility to be fired coincidentally by random noise hits. Therefore, trigger logic is adopted to select real shower events. The trigger logic of KM2A has been sufficiently explored and more details could be found elsewhere [可. In this simulation, two choices are provided for the trigger, one is only using the time window and another could use both time and space windows. The windows and threshold are flexibly set by reading external parameter file. Once survive the trigger logic, all the hits including shower hits and noise hits within a window of 10 us center on the start time of trigger window will be output as an event. This is same as the practical process. It is worth to note that, at least three shower hits are required to apply the trigger logic. Hence, this simulation doesn't simulate pure noise event due to coincident noise hits.

\section{Comparison with partial array data}

A partial KM2A array with 33 EDs was operated since February 2018 at daocheng site. This partial array was enlarged to 71 EDs and 10 MDs in December 2018, which was changed to 99 EDs and 8 MDs in April 2019. These partial arrays are all operated by requiring the number of fired EDs to be at least 5 within a time window of $200 \mathrm{~ns}$. Here the simulation sample of 71EDs+10MDs array are compared with the experiment data collected in 16th February 2019 for the number of particles and muons. For comparison the experiment data collected in 2nd June 2019 with a configure of 99EDs+8MDs are also presented in the Figure 5. In this simulation, the Gaisser model with 5 cosmic ray compositions are used [ $[$ ]. According to the Figure 5, the number of particles recorded by ED array and the number of muons recorded by MD array are generally consistent with the simulation results. The trigger rate estimated by $\mathrm{MC}$ is about $10 \%$ lower than the data, which is mainly from the events with a few particles according to Figure 5. The distribution of muon number also shows some difference between MC and data at number greater than 100, which may be due to an electronics problem. A good agreement is achieved after solving the problem according to the data in 2nd June 2019. The ratio of muon number and electromagnetic particle number is a key parameter for KM2A to discriminate $\gamma$-ray from the cosmic ray background. This parameter also shows a good agreement between data and simulation according to Figure 5. These results general verify the reliability the simulation code G4KM2A. 

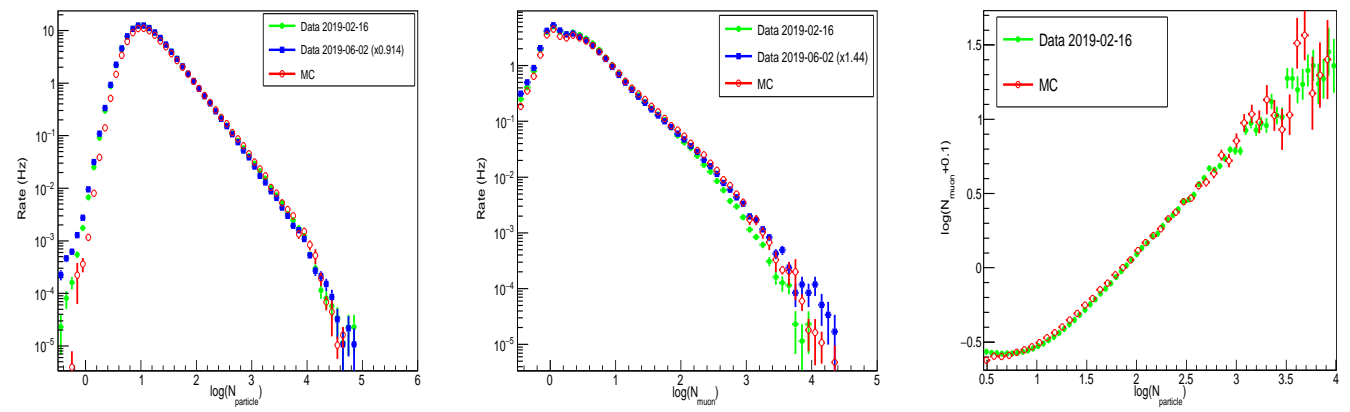

Figure 5: Comparison of the experiment data with the simulation. Left: number of particles detected by ED array. Middle: number of muons detected by MD array. Right: number of ED particles vs number of MD muons. To match the events rate in 16th February 2019, the event rate in 2nd June 2019 is rescaled with a factor.

\section{Summary}

In summary, simulation of KM2A detector has developed within Geant4 package, which is named as G4KM2A. This code can simulate the KM2A array with any configuration. To overcome the memory overflow, the simulation process flow is adjusted by reading-in the particles of shower one by one in the particle generator. This experience can be adopted by other similar large detector simulation in the further. Some simplifications are used to speed up the simulation by a factor of at least 30 times, which satisfy the practical requirement. The number of electromagnetic particles recorded by ED array and the number of muons recorded by MD array yielded by the simulation are consistent with the partial KM2A array data, which verify the reliability the simulation.

\section{References}

[1] He, H.H., for the LHAASO Collaboration, 2018, Radiation Detection Technology and Methods 2, 7

[2] Liu, Y., Cao, Z., Chen, S.Z., et al. 2016, ApJ, 826, 63

[3] Cui, S.W., Liu, Y., Liu, Y.J., Ma, X.H., 2014, Astroparticle Physics 54, 86

[4] Heck, D., Knapp, J., Capdevielle, J. N., Shatz, G., \& Thouw, T. 1998, Forschungszentrum Karlsruhe Report No. FZKA 6019

[5] Agostinelli, S., Allison, J., Amako, K., et al., 2003, Nuclear Instruments and Methods in Physics Research Section A, 506, 250

[6] Wu, S., Chen, L., Chen, S.Z., et al. 2018, Astroparticle Physics, 103, 41

[7] Gaisser, T.K., 2012, Astroparticle Physics, 35, 801 